\title{
On a Characterization of Zero-Inflated Negative Binomial Distribution
}

\author{
R. Suresh', G. Nanjundan², S. Nagesh'1, Sadiq Pasha ${ }^{2}$ \\ ${ }^{1}$ Department of Statistics, Karnatak University, Dharwad, India \\ ${ }^{2}$ Department of Statistics, Bangalore University, Bangalore, India \\ Email: sureshramaiah@gmail.com, nanzundan@gmail.com, nageshstatistics@gmail.com, \\ sadiqiz1991@gmail.com
}

Received 23 August 2015; accepted 9 October 2015; published 13 October 2015

Copyright (C) 2015 by authors and Scientific Research Publishing Inc.

This work is licensed under the Creative Commons Attribution International License (CC BY). http://creativecommons.org/licenses/by/4.0/

(c) (i) Open Access

\section{Abstract \\ Zero-inflated negative binomial distribution is characterized in this paper through a linear diffe- rential equation satisfied by its probability generating function.}

\section{Keywords}

Zero-Inflated Negative Binomial Distribution, Probability Distribution, Probability Generating Function, Linear Differential Equation

\section{Introduction}

Zero-inflated discrete distributions have paved ways for a wide variety of applications, especially count regression models. Nanjundan [1] has characterized a subfamily of power series distributions whose probability generating function (pgf) $f(s)$ satisfies the differential equation $(a+b s) f^{\prime}(s)=c f(s)$, where $f^{\prime}(s)$ is the first derivative of $f(s)$. This subfamily includes binomial, Poisson, and negative binomial distributions. Also, Nanjundan and Sadiq Pasha [2] have characterized zero-inflated Poisson distribution through a differential equation. In the similar way, Nagesh et al. [3] have characterized zero-inflated geometric distribution. Along the same lines, zero-inflated negative binomial distribution is characterized in this paper via a differential equation satisfied by its pgf.

A random variable $X$ is said to have a zero-inflated negative binomial distribution, if its probability mass function is given by

$$
p(x)= \begin{cases}\varphi+(1-\varphi) p^{r} & x=0 \\
(1-\varphi)\left(\begin{array}{c}
x+r-1 \\
x
\end{array}\right) p^{r} q^{x} & x=1,2, \cdots,\end{cases}
$$


where $0<\varphi<1,0<p<1, \quad p+q=1$, and $r>0$.

The probability generating function of $X$ is given by

$$
\begin{aligned}
f(s) & =E\left(s^{X}\right)=\sum_{x=0}^{\infty} p(s) s^{x}, 0<s<1 \\
& =\varphi+(1-\varphi) p^{r} \sum_{x=0}^{\infty}\left(\begin{array}{c}
x+r-1 \\
x
\end{array}\right) q^{x} \\
f(s) & =\varphi+(1-\varphi) \frac{p^{r}}{(1-q s)^{r}} .
\end{aligned}
$$

Hence the first derivative of $f(s)$ is given by

$$
f^{\prime}(s)=(1-\varphi) r q \frac{p^{r}}{(1-q s)^{r+1}} .
$$

\section{Characterization}

The following theorem characterizes the zero-inflated negative binomial distribution.

Theorem 1 Let $X$ be a non-negative integer valued random variable with $0<P(X=0)<1$. Then $X$ has a zero-inflated negative binomial distribution if and only if its pgf $f(s)$ satisfies

$$
f(s)=a+b(1+c s) f^{\prime}(s) \text {, }
$$

where $a, b, c$ are constants.

Proof. 1) Suppose that $X$ has zero-inflated negative binomial distribution with the probability mass function specified in (1). Then its pgf can be expressed as

$$
\begin{aligned}
& f(s)=\varphi+(1-\varphi) \frac{r q p^{r}(1-q s)}{r q(1-q s)^{r+1}} \\
& f(s)=\varphi+\frac{1}{r q}(1-q s) f^{\prime}(s) .
\end{aligned}
$$

Hence $f(s)$ in (4) satisfies (3) with $a=\varphi, b=1 / r q, c=-q$.

2) Suppose that the pgf of $X$ satisfies the linear differential equation in (3).

Writing the Equation (3) as

$$
y=a+b(1+c x) \frac{\mathrm{d} y}{\mathrm{~d} x},
$$

we get

$$
\frac{\mathrm{d} y}{y-a}=\frac{1}{b c} c \frac{\mathrm{d} x}{1+c x} .
$$

On integrating both sides w.r.t. $x$, we get

$$
\begin{aligned}
& \int \frac{\mathrm{d} y}{y-a}=\frac{1}{b c} \int \frac{c \mathrm{~d} x}{1+c x} \\
& \Rightarrow \log (y-a)=\frac{1}{b c} \log (1+c k)+k_{1} \text {, where } k_{1} \text { is an arbitary constant. } \\
& \therefore \log (y-a)=\log (1+c k)^{\frac{1}{b c}}+\log k \text {, by writing } k_{1}=\log k \\
& =\log \left[k(1+c k)^{\frac{1}{b c}}\right]
\end{aligned}
$$

That is

$$
y=a+k(1+c x)^{\frac{1}{b c}} .
$$


The solution of the differential equation in (3) becomes

$$
f(s)=a+k(1+c s)^{\frac{1}{b c}} .
$$

If either $b$ or $c$ or both are equal to zero, then $\frac{1}{b c}=\infty$ and hence $f(s)$ has no meaning. Thus, both $b$ and $c$ are non-zero. Since $f(s)$ is a pgf, it is a power series of the type $p_{0}+p_{1} s+p_{2} s^{2}+\cdots$. When either $c>0$ or $\frac{1}{b c}$ is not a negative integer, the expansion of the factor $(1+c s)^{\frac{1}{b c}}$ on the right hand side of (5) will have negative coefficients, which is not permissible because $f(s)$ is a pgf. Hence the equation in (5) can be written as

$$
f(s)=a+k(1-d s)^{-N},
$$

where $N$ is a positive integer. Since $f(1)=1, k=(1-a)(1-d)^{N}$.

Therefore

$$
f(s)=a+(1-a)(1-d)^{N}(1-d s)^{-N} .
$$

Hence $f(s)$ in (6) satisfies (2) with $a=\varphi, p=(1-d), q=d$, and $N=r$.

This completes the proof of the theorem.

Also, it can be noted that when $N=r=1$, the negative binomial distribution reduces to geometric distribution and the Theorem 1 in Section 2 concurs with the characterization result of Nagesh et al. [3].

\section{References}

[1] Nanjundan, G. (2011) A Characterization of the Members of a Subfamily of Power Series Distributions. Applied Mathematics, 2, 750-751. http://dx.doi.org/10.4236/am.2011.26099

[2] Nanjundan, G. and Pasha, S. (2015) A Note on the characterization of Zero-Inflated Poisson Distribution. Open Journal of Statistics, 5, 140-142. http://dx.doi.org/10.4236/ojs.2015.52017

[3] Nagesh, S., Nanjundan, G., Suresh, R. and Pasha, S. (2015) A Characterization of Zero-Inflated Geometric Model. International Journal of Mathematics Trends and Technology, 23, 71-73. http://dx.doi.org/10.14445/22315373/IJMTT-V23P510 